\title{
Interplay of nonlinearity and geometry in a DNA-related, Klein-Gordon model with long-range dipole-dipole interaction
}

\section{Archilla, J. F.R.; Christiansen, Peter Leth; Gaididei, Yuri Borisovich}

Published in:

Physical Review E. Statistical, Nonlinear, and Soft Matter Physics

Link to article, DOI:

10.1103/PhysRevE.65.016609

Publication date:

2002

Document Version

Publisher's PDF, also known as Version of record

Link back to DTU Orbit

Citation (APA):

Archilla, J. F. R., Christiansen, P. L., \& Gaididei, Y. B. (2002). Interplay of nonlinearity and geometry in a DNArelated, Klein-Gordon model with long-range dipole-dipole interaction. Physical Review E. Statistical, Nonlinear, and Soft Matter Physics, 65(1), 016609. https://doi.org/10.1103/PhysRevE.65.016609

\section{General rights}

Copyright and moral rights for the publications made accessible in the public portal are retained by the authors and/or other copyright owners and it is a condition of accessing publications that users recognise and abide by the legal requirements associated with these rights.

- Users may download and print one copy of any publication from the public portal for the purpose of private study or research.

- You may not further distribute the material or use it for any profit-making activity or commercial gain

- You may freely distribute the URL identifying the publication in the public portal 


\title{
Interplay of nonlinearity and geometry in a DNA-related, Klein-Gordon model with long-range dipole-dipole interaction
}

\author{
J. F. R. Archilla \\ Department of Applied Physics I, University of Sevilla, Avenida Reina Mercedes s/n, 41012-Sevilla, Spain \\ P. L. Christiansen \\ Informatics and Mathematical Modelling, The Technical University of Denmark, DK-2800 Lyngby, Denmark
}

Yu. B. Gaididei

Bogolyubov Institute for Theoretical Physics, 03143 Kiev, Ukraine

(Received 12 July 2001; published 21 December 2001)

\begin{abstract}
Most of the studies on mathematical models of DNA are limited to next neighbor interaction. However, the coupling between base pairs is thought to be caused by dipole interaction, and, when the DNA strand is bent, the distances between base pairs become shorter, therefore the interactions with distant base pairs have to be taken into account. In this paper we analyze the existence and stability of breathers, i.e., localized oscillations in a simple model of bent DNA with long-range dipole interaction. Breathers have been suggested as precursors of the denaturation bubble.
\end{abstract}

DOI: 10.1103/PhysRevE.65.016609

PACS number(s): 63.20.Ry, 63.20.Pw, 63.50.+x, 87.10.+e

\section{INTRODUCTION}

During the last years a great deal of attention has been paid to localized oscillations in nonlinear discrete systems, which are usually known as discrete breathers, to differentiate them from the entity with the same name in continuous systems. In a basic mathematical paper by Mackay and Aubry [1], their existence and stability were established under rather general conditions. This mathematical proof, based on the limit with no coupling, has become one of the starting points to obtain breathers, as "exact" numerical solutions, up to machine precision [2-5]. A thorough review can be found in Ref. [6].

Many researchers have worked in the extension of the proofs to more general conditions, applications to specific fields and different systems, and to obtain experimental evidence of their existence in real systems [7-12].

A particularly interesting real system is DNA, where localization of the energy $[13,14]$ has been suggested as a precursor of the transcription bubble [15], and where moving localized oscillations can be the way for transporting information [16] or for explaining secondary breaks, when DNA is irradiated with ionizing radiation [17]. A great number of different linear and nonlinear models has been proposed for DNA, according to the phenomena that are intended to explain Ref. [18]. An initially simple model, that has been progressively complicated, is the Peyrard-Bishop model [19], where the dynamics of DNA is reduced to a Klein-Gordon system, the variables being the distances between nucleotides within each base pair. This is a natural field for discrete breathers, as the disorder or inhomogeneity of the double strand is not an obstacle either to the mathematical proof or to the numerical methods, and where fundamental questions arise on the interplay of nonlinearity and inhomogeneity [20-22].
The important and related point we address here is the influence of geometry, i.e., of the shape of the double strand, on the existence and stability of breathers, and therefore on localization of energy. Geometry is not relevant, in the framework of the Peyrard-Bishop model, as long as only nearest neighbor interaction is taken into account. However, one of the two hydrogen bonds that is responsible for the interbase coupling, the hydrogen bond in the $N-H \cdots 0$ group, is characterized by a finite dipole moment. Therefore a stretching of the base pair will produce a change of the dipole moment and an interaction between dipoles, with a dependence on the distance $r$ between base pairs as $1 / r^{3}$, takes place. With the long-range interaction the shape of the molecule becomes critical and cannot be neglected any longer.

Bending of DNA has been studied in several papers. In Ref. [13] the bending is introduced as an inhomogeneity. Perturbative methods and numerical simulation is used. In Ref. [23], the bending is introduced as a change of the coupling constant due to the fact that the bases become closer inside the bend. Numerical simulation is also used. Longrange interaction has been taken in account in bent DNA models using the nonlinear Schrödinger (NLS) equation $[24,25]$. However, the effect of curvature and long-range interaction in a DNA model has not yet been addressed for exact breathers.

We choose a model as simple as possible to isolate the effect of the bending and the long-range interaction. Therefore we restrict the shape to a parabola in a plane, the dipole moments being perpendicular to it and parallel among them, not taking into account the angles between them, the helicity of the molecule, and the stretching. The sketch of the model is shown in Fig. 1. An adequate choice of units of time and distance in the different directions allow us to eliminate all parameters except for the coupling and the curvature. 


\section{MODEL}

Our model is described by the Hamiltonian

$$
H=\sum_{n=1}^{N}\left(\frac{1}{2} \dot{u}_{n}^{2}+\frac{1}{2} \omega_{0}^{2} u_{n}^{2}+\frac{1}{4} s u_{n}^{4}+\frac{1}{2} \varepsilon \sum_{m=1}^{N} J_{n m} u_{n} u_{m}\right) \text {. }
$$

The variables $u_{n}=u_{n}(t)$ represent the separation of the nucleotides in a base pair with respect to their equilibrium distance. The on-site potential corresponds thus to the hydrogen-bridge bond between nucleotides. It is composed of a linear term $1 / 2 \omega_{0}^{2} u_{n}^{2}$, and a nonlinear term $\frac{1}{4} s u_{n}^{4}$, where $s$ is a parameter that tunes the nonlinearity. If $s$ is positive the on-site potential is hard, and soft otherwise.

The last term is the long-range dipole interaction, $J_{n m}$ being the coupling constants between base pairs, with values, for $n \neq m, J_{n m}=1 /\left|\vec{r}_{n}-\vec{r}_{m}\right|^{3}$, and 0 otherwise. We suppose that the dipole moments are parallel among them and orthogonal to the plane where the bending takes place.

The vectors $\vec{r}_{n}=\left(x_{n}, y_{n}\right)$ describe the positions of the base pairs in a two-dimensional space where the system is embedded. They do not depend on time as we suppose that the shape of the system is constant in time. This shape is supposed to be a parabola, which can be an approximation in the neighborhood of the bending point, i.e., $y_{n}=1 / 2 \kappa x_{n}^{2}$, where $\kappa$ is the curvature of the parabola.

The time is scaled, so, as $\omega_{0}=1$, the distance between adjacent base pairs is scaled to be the unity, i.e., $\left|\vec{r}_{n}-\vec{r}_{n+1}\right|$ $=1$. The distance between nucleotides, being perpendicular to $\vec{r}_{n}$, can be scaled independently, so that the nonlinear term can be written $\pm 1 / 4 u_{n}^{4}$.

Therefore there are only two parameters in the system, the curvature $\kappa$ and $\varepsilon$, which represents the strength of the coupling, or, in other words, the relation between the coupling energy and the on-site energy.

The dynamical equations become

$$
\ddot{u}_{n}+\omega_{0}^{2} u_{n}+s u_{n}^{3}+\varepsilon \sum_{m=1}^{N} J_{n m} u_{m}=0, \quad n=1 \cdots N .
$$

\section{METHOD}

We look for breathers, that is, localized, time-reversible, periodic solutions of Eq. (2). The procedure consists of expressing the functions $u_{n}$ as truncated cosine Fourier series of specified frequency $\omega_{\mathrm{b}}$, that is, $u_{n}=z_{0, n}$ $+\sum_{k=1}^{k_{m}} 2 z_{k, n} \cos \left(k \omega_{\mathrm{b}} t\right)$, and collecting together the coefficients corresponding to different $k$. Then, we obtain a system of algebraic equations on $z_{k, n}$. Starting from the anticontinuous limit, i.e., $\varepsilon=0$, where we have solutions for the isolated variables, we can continue them using the Newton method up to some unspecified value of $\varepsilon$. The time reversibility condition allows us to eliminate the trivial time shift degeneracy. The theorem of existence can be found in the so called basic mathematical paper, Ref. [1], and a thorough description of the numerical methods can be found in Refs. $[2,3,5]$. The linear stability is checked by studying the eigenvalues of

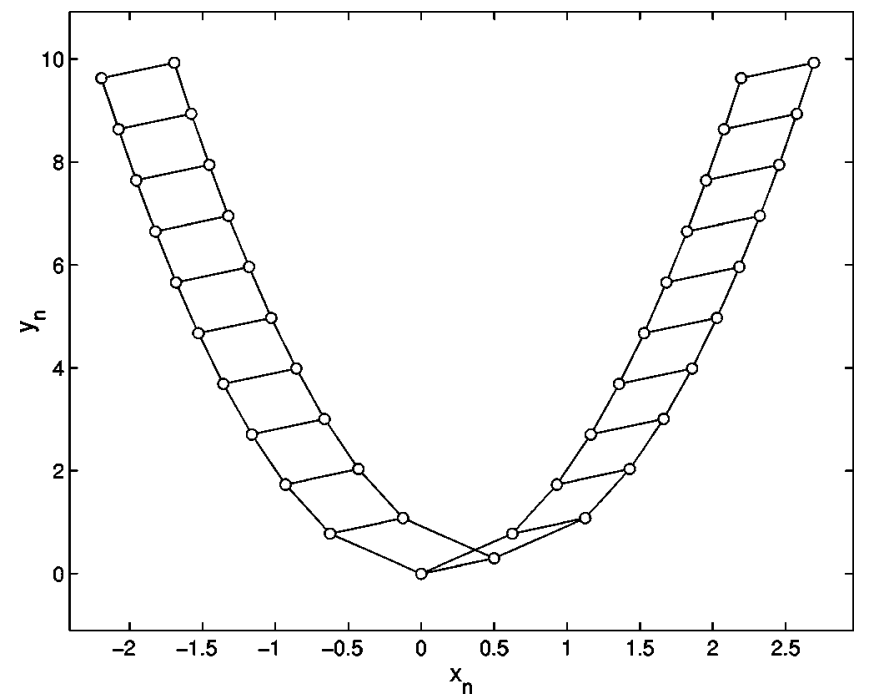

FIG. 1. Sketch of the model. The circles represent the nucleotides.

the monodromy $[4,26]$, i.e., the matrix $M_{0}$, given by

$$
\left[\left\{\xi_{n}(T)\right\},\left\{\dot{\xi}_{n}(T)\right\}\right]=M_{0}\left[\left\{\xi_{n}(0)\right\},\left\{\dot{\xi}_{n}(0)\right\}\right],
$$

where $T=2 \pi / \omega_{\mathrm{b}}$, is the period of the breather, and $\left[\left\{\xi_{n}(t)\right\},\left\{\xi_{n}(t)\right\}\right]$ is the column matrix of positions and velocities of the solutions of the linearization of Eq. (2), that is, the result of the numerical integration ${ }^{1}$ of the following equations:

$$
\begin{gathered}
(N(u(t), \varepsilon) \cdot \xi)_{n} \equiv \ddot{\xi}_{n}+\omega_{0}^{2} \xi_{n}+s u_{n}^{3} \xi_{n}+\varepsilon \sum_{m=1}^{N} J_{n m} \xi_{m}=0 \\
n=1 \cdots N .
\end{gathered}
$$

Here $\xi=\left\{\xi_{n}(t)\right\}$ represent the perturbations of the breather solution, and are not restricted to time reversible functions. The operator $N(u(t), \varepsilon) \equiv\left\{N_{n}(u(t), \varepsilon)\right\}$ is called the Newton operator. Therefore the solutions of Eq. (4) can be described as the eigenvectors of $N$ corresponding to the zero eigenvalue.

As the linearized system is real and Hamiltonian, the monodromy is also a real and symplectic operator. This implies that if $\lambda$ is an eigenvalue of $M_{0}$, so are $\lambda^{*}, 1 / \lambda, 1 / \lambda^{*}$. Stability means that all the eigenvalues have modulus 1 . The fact that the derivative of the breather $\left\{\dot{u}_{n}(t)\right\}$ is a solution of Eq. (4) brings about that there is always a double eigenvalue at $1+0 i$.

\section{LINEAR MODES}

To start with, we study the linear modes of the system. In Fig. 2 are represented the frequencies of the linear modes for

\footnotetext{
${ }^{1} \mathrm{~A}$ symplectic integrator is highly recommended to get accurate results [27].
} 

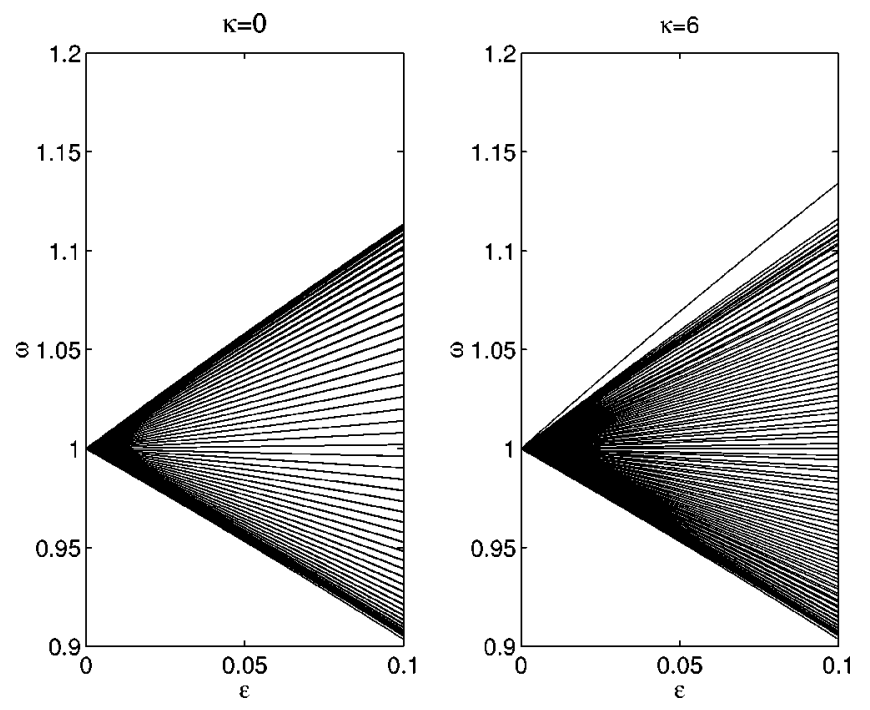

FIG. 2. Frequencies of the linear modes with respect to the coupling parameter $\varepsilon$ for curvatures $\kappa=0$ and $\kappa=6$.

a system of 101 particles as a function of the coupling parameter $\varepsilon$, for two different values of $\kappa$.

The degree of localization can be measured with the function

$$
\phi(u)=\frac{\sum_{n=1}^{N} u_{n}^{4}}{\left(\sum_{n=1}^{N} u_{n}^{2}\right)^{2}}
$$

or its inverse which gives a measure of the number or variables involved in the mode. Therefore $\phi=1 / N$, when all the variables have the same amplitude, $\phi=1 / n$, when $n$ variables have the same amplitudes, and the rest are zero.

Several features emerge from the analysis of the linear modes, their degree of localization, and by plotting them.

(i) For the straight line, $\kappa=0$, all the modes are delocalized, except for one at the bottom, localized at both ends of the chain, due to the finite size of the system. This mode, unless stated otherwise, will not be taken in account hereafter.

(ii) For $\kappa \neq 0$, a localized symmetric mode separates from the top of the band.

(iii) Above a threshold value of $\kappa \approx 8$, another localized asymmetric mode separates from the bottom of the band. The origin of this mode is clear as this value of $\kappa$ is slightly above the value when the distance between the two closest particles to the bending point becomes smaller than unity. Therefore their interaction is stronger that the one with their neighbors.

Figure 3 shows the splitting of these two eigenvalues when $\kappa$ is changed at constant $\varepsilon$.

As it can be observed in this figure, the spreading of the linear band with curvature at constant coupling is minimal except for the localized modes that segregate from it.
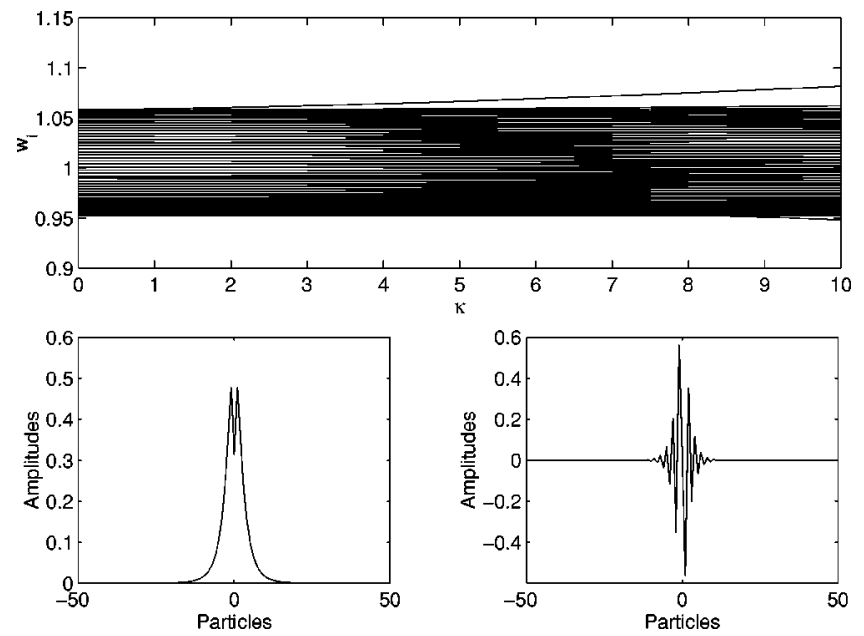

FIG. 3. Top: frequencies of the linear modes with respect to the curvature. $\kappa$, for fixed coupling $\varepsilon=0.05$. The maximum corresponds to wave number $q=0$ and the minimum to $q=\pi$. Bottom left and right: profiles of the localized modes at the top and the bottom of the band, respectively. The calculations have been done with 101 oscillators.

\section{ONE-SITE BREATHER WITH HARD POTENTIAL}

The frequency of the one-site breather with hard potential, i.e., with $s=1$, in Eq. (1), has to be greater than the linear frequency $\omega_{0}=1$, thus we have chosen $\omega_{\mathrm{b}}=1.1$, so that the nonlinear effects are significant but, on the other hand, not too strong, as the nonlinearities in DNA are thought to be weak.

The term one-site breather refers to the situation at the start of the path continuation, i.e., at zero coupling, because, as soon as this is switched on, other sites are involved and even the main amplitude can change position in the chain.

We have studied what we call the central breather, the lateral breather, and the second breather, i.e., located at zero coupling at the bending point, at the nearest neighbor, and at the next nearest neighbor.

\section{A. Central breather evolution}

We denote the dynamical equations Eq. (2) as $f(u)=0$, emphasizing when it is convenient the dependence on a parameter as, for example, $f(u, \varepsilon)=0$. Equally, we use the same notation for the equivalent function in the Fourier space, i.e., $f(z)=0$, or $f(z, \varepsilon)=0$, and so on. The continuation is done in the Fourier space, so that, if $f\left(z_{0}, \varepsilon_{0}\right)=0$, and $\varepsilon_{1}=\varepsilon_{0}+\delta \varepsilon$ is a value close to $\varepsilon_{0}$, and $z_{1}=z_{0}+\delta z$ is its corresponding solution, then

$$
f\left(z_{0}+\delta z, \varepsilon_{1}\right) \approx f\left(z_{0}, \varepsilon_{1}\right)+\partial_{z} f\left(z_{0}, \varepsilon_{0}\right) \delta z=0 .
$$

Therefore, from this equation, the value of $\delta z$ can be obtained, provided that the Jacobian $\partial_{z} f\left(z_{0}, \varepsilon_{0}\right)$ is invertible. The procedure can be repeated until $f\left(z_{1}, \varepsilon_{1}\right)=0$ up to machine precision. If the Jacobian is not invertible, that is, if one of its eigenvalues is zero, the conditions for the implicit function theorem are not satisfied, therefore a bifurcation can take place. Of course, the numerical methods do not allow us 


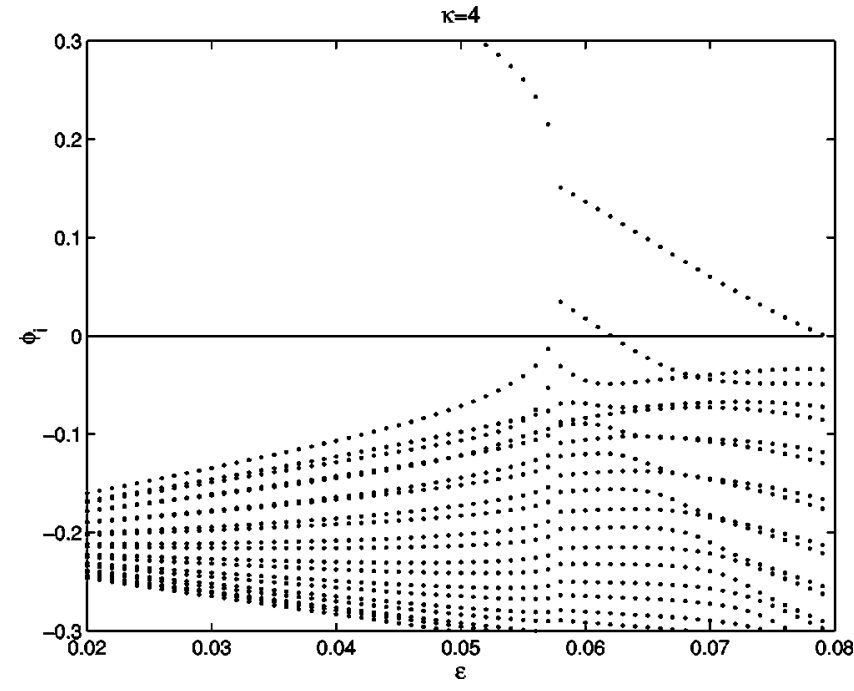

FIG. 4. Evolution of the Jacobian eigenvalues $\phi_{i}$ for the central breather. An eigenvalue approaches zero and another branch of solutions is taken.

to find an exact zero eigenvalue, but this point can be detected by the slowness or lack of convergence of the Newton method, and by observation of the evolution of the Jacobian eigenvalues as the parameter is changed.

Care has to be taken, as the Newton method, in the vicinity of a bifurcation, may jump often to another branch of solutions in the neighborhood of the continued one. We can sometimes continue a path across a bifurcation by adding to the breather a fraction of the eigenvector corresponding to the nearly zero eigenvalue, scaling, and starting the Newton method again.

Figure 4 shows a zoom of the evolution of the Jacobian eigenvalues. The first zero crossing corresponds to a bifurcation where the breather ceases to exist and jumps to a close branch. The second zero crossing is related with the change of stability of the breather as it can be understood by Aubry's band theory [4] but it will be too long to explain here.

The band of eigenvalues below zero corresponds to the first harmonic phonon band. An eigenvalue can be seen separating from it and approaching to zero bringing about the bifurcation. This mode is the double humped mode (DHM), similar to the one found at the top of the linear band.

The first positive branch of eigenvalues corresponds to what will be called the breather mode (BM), as it has no related linear mode and has often the same profile as the breather. In systems with hard potential the breather mode appears above zero as the frequency of the breather increases with its growth. The opposite happens with soft potentials. This mode produces eventually a third bifurcation, where the breather disappears.

These two modes are going to be present for almost any value of the parameters, but different patterns of bifurcation are going to happen depending on the value of the coupling parameter. We better describe the evolution in the parameter space $(\kappa, \varepsilon)$, keeping $\varepsilon$ fixed and varying $\kappa$ as it has more physical meaning, corresponding to the process of bending the chain. We also limit, in general, the values of curvature to
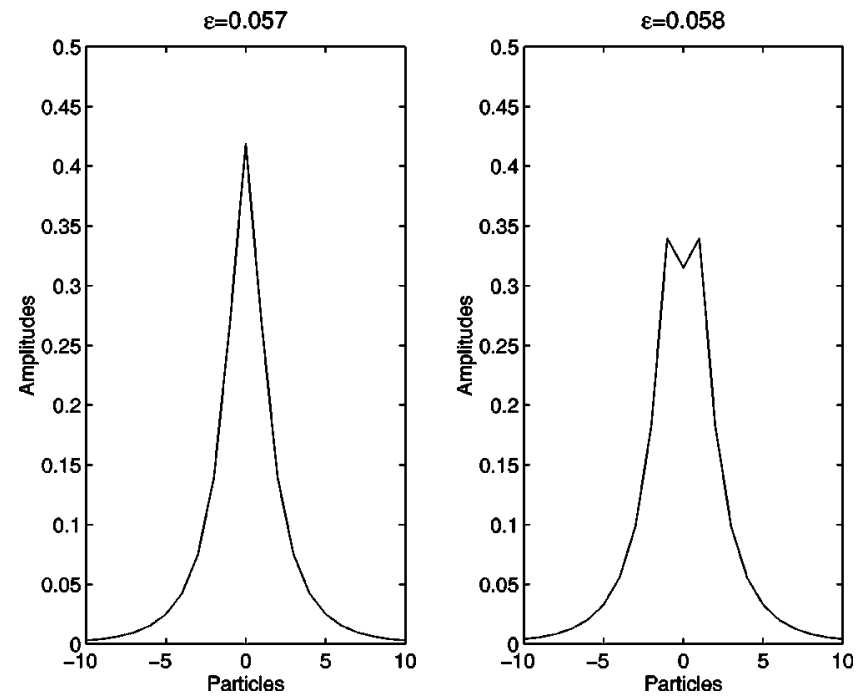

FIG. 5. Profiles of the central breather just before and after the bifurcation for $\kappa=4$; the bending point is at the particle 0 .

something over $\kappa_{c}=4 \sqrt{3} \approx 7$, as this is the value above which the two neighbors of the bending point become closer than the interparticle distance, and we believe this is not expected to happen in real systems. Therefore expressions like low or intermediate curvature have to be understood compared to $\kappa_{c}$.

There are three basic scenarios:

(i) For low coupling $(\varepsilon \leqq 0.03)$, no bifurcation happens when increasing the curvature (it will happen for values of curvature around 15 with the DHM). That is, the centered breather $(\mathrm{CB})$ exists. We are in the nonlinear range and the breather feels very weakly the shape of the molecule.

(ii) For intermediate coupling $(0.03 \leq \varepsilon \leq 0.07)$, a bifurcation occurs when the DHM collides with the CB at $\kappa \approx 6.2$ (3.5 for $\varepsilon=0.06$ ), after a small gap (smaller with increasing $\varepsilon)$, the double humped breather (DHB) appears and it will collide with the $\mathrm{BM}$ for values of curvature around 11 . The centered breather exists for low to intermediate curvature, and then the DHB takes command.

(iii) For large coupling $(\varepsilon \gtrsim 0.07)$, there is an evolution where the CB evolves continuously to the DHB without bifurcation, passing through a state where the profile of the breather is flat at the top, with three oscillators with the same amplitude.

Figure 5 shows the profiles of the central breather before and after the first bifurcation.

\section{B. Central breather stability}

The breather becomes unstable slightly before this first bifurcation (at $\varepsilon=0.057$ ), i.e., at $\varepsilon=0.054$, and continues to be unstable until the second zero crossing takes place. This is shown in Fig. 6.

This is a general picture with the appearance of the bubble of stability shown in the top figure, slightly before the bifurcation. Afterwards the breather becomes stable again before its definitive destruction. 

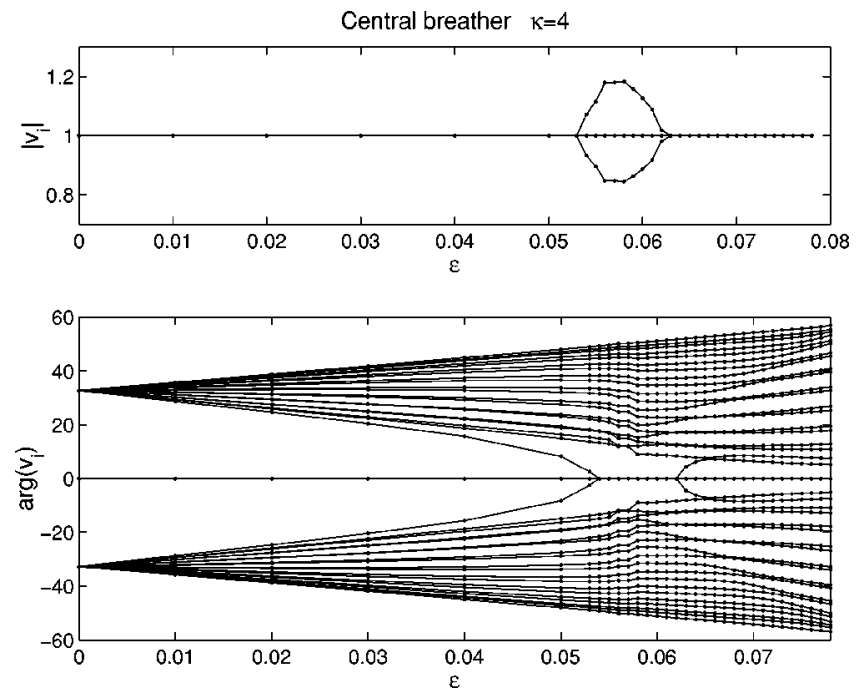

FIG. 6. Evolution of the monodromy eigenvalues with the coupling $\varepsilon$, for the central breather. Top: modulus; bottom: arguments. The points are the calculated eigenvalues, the lines are guides to the eye.

\section{Lateral breather and symmetry breaking}

We refer in this way to the breather located at $\varepsilon=0$ at the nearest neighbor of the bending point (hereafter named as the first neighbor). Continuation of this breather with the coupling $\varepsilon$ leads to an asymmetric breather with its main amplitude located at this point. Eventually, it experiences a bifurcation bringing about the same symmetric double humped breather as in the previous case. The same three different regimes of low, intermediate, and large coupling are also found.

Figure 7 shows the profiles at two points just before the bifurcation and after this has taken place, where the breather is again a symmetric one.

It should be noted that the asymmetric lateral breather (ALB) ceases to exist exactly when the central breather be-
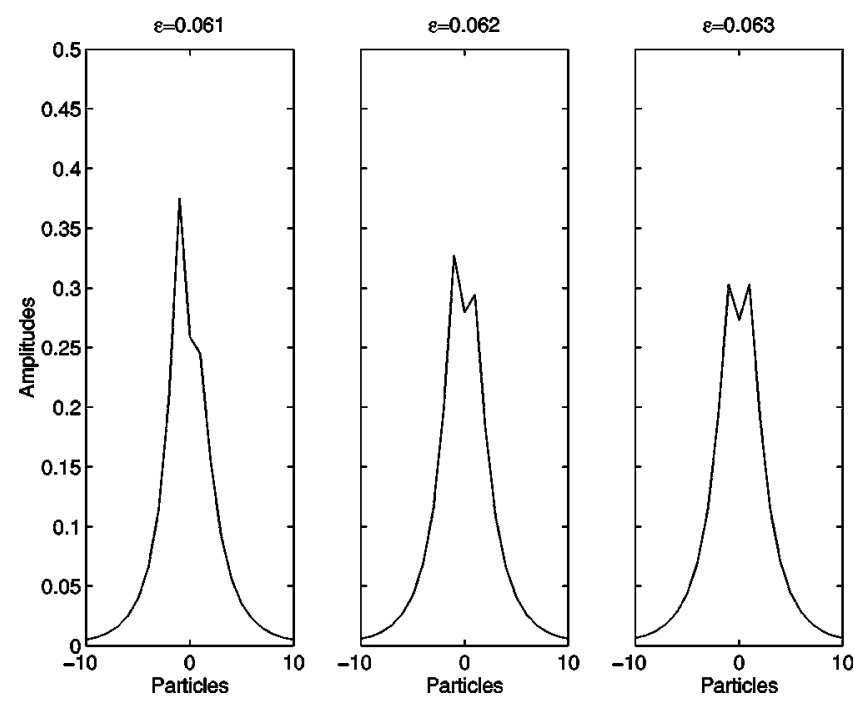

FIG. 7. Lateral breathers for $\kappa=4$. Left and center: before the bifurcation; right: afterwards.
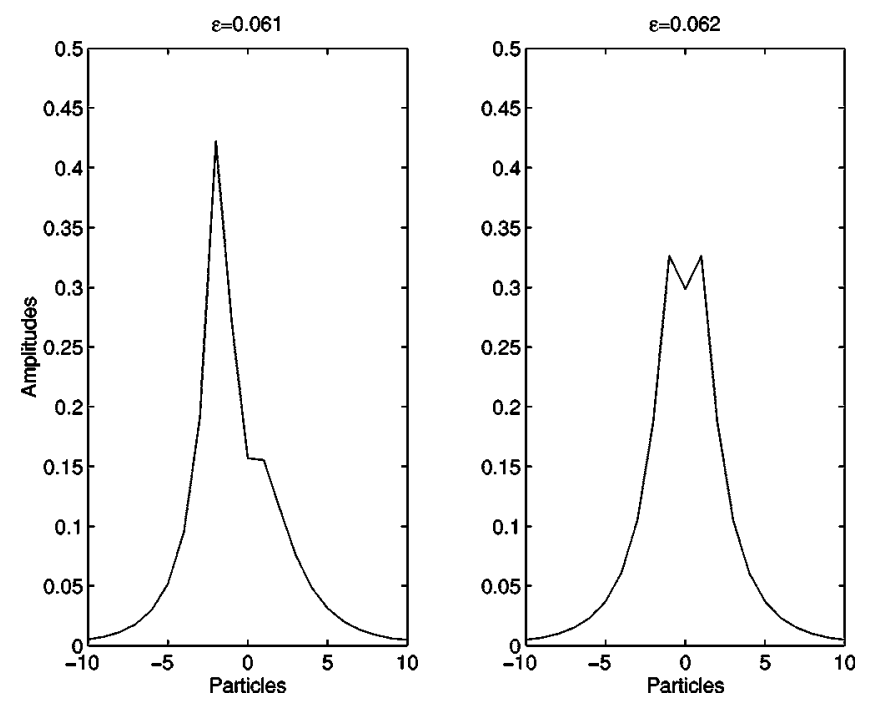

FIG. 8. Second neighbor breathers for $\kappa=4$. Left: before the bifurcation; right: afterwards.

comes stable again. Observation of the instability eigenvector of the central breather and simulations with a slight perturbation in that direction confirm that it tends to evolve to the ALB.

Therefore we obtain that in a intermediate region of the parameters the nonlinearity is able to maintain an asymmetric state, which disappears with strong enough coupling. The lateral breather acts as an attractor for the central one at intermediate coupling/curvature. Due to the reflection symmetry of the system both lateral breathers have the same properties. The consequence is that we are in the presence of an inverse pitchfork bifurcation with symmetry breaking.

\section{Second neighbor}

Similar results are obtained when the continuation is done with the breather initially located at the second neighbor of the bending point. Again, a bifurcation occurs bringing about the symmetric, double humped breather. The profiles of the breathers before and after the bifurcation are shown in Fig. 8.

\section{CONCLUSIONS}

We address the problem of existence and stability of breathers, i.e., periodic localized solutions, on a model for DNA with dipole long-range interaction (LRI). The LRI brings about that the properties of the system are sensitive to the shape of the system. We limit the shape to a parabola and constrain the orientation of the dipoles to a direction normal to the plane of the parabola, in order to isolate the effect of the curvature. We study the path of the breathers to their annihilation.

We find that there is a symmetric, double humped mode as the dominant one for strong enough coupling. At intermediate coupling, there exist stable asymmetric breathers which become unstable and stable again through inverse pitchfork bifurcations with symmetry breaking.

There are two competing sources of localization, the inhomogeneity due to the bending and the LRI, and the non- 
linearity. The relationship between the nonlinear energy of the hydrogen bonds and the dipole interaction in DNA is not well known. If it is high enough a much larger variety of breathers will exist and be stable in the neighborhood of a bending, while, in the opposite case, only an almost linear breather, symmetric and double humped, will exist. In this case, the breathers at bending points could play a decisive role as a precursor of the denaturation bubble. It is interesting that a model with attractive long-range interaction shows also localization at the bending points through a different mechanism [28].

\section{ACKNOWLEDGMENTS}

This work has been supported by the European Union under the RTN project LOCNET, HPRN-CT-1999-00163. Two of the authors, J. F. R. Archilla and Yu. B. Gaididei, acknowledge Informatics and Mathematical Modelling for hospitality.
[1] R. MacKay and S. Aubry, Nonlinearity 7, 1623 (1994).

[2] J. Marín and S. Aubry, in Nonlinear Klein-Gordon and Schrödinger Systems: Theory and Applications, edited by L. Vázquez, L. Streit, and V. Pérez-García (World Scientific, Singapore, 1995), pp. 317-323.

[3] J. Marín and S. Aubry, Nonlinearity 9, 1501 (1996).

[4] S. Aubry, Physica D 103, 201 (1997).

[5] J. Marín, S. Aubry, and L.M. Floría, Physica D 113, 283 (1998).

[6] S. Flach and C. Willis, Phys. Rep. 295, 181 (1998).

[7] B. Swanson et al., Phys. Rev. Lett. 82, 3288 (1999).

[8] F.M. Russell, Y. Zolotaryuk, J.C. Eilbeck, and T. Dauxois, Phys. Rev. B 55, 6304 (1997).

[9] U.T. Schwarz, L.Q. English, and A.J. Sievers, Phys. Rev. Lett. 83, 223 (1999).

[10] P. Binder et al., Phys. Rev. Lett. 84, 745 (2000).

[11] E. Trias, J.J. Mazo, and T.P. Orlando, Phys. Rev. Lett. 84, 741 (2000).

[12] P. Binder, D. Abraimov, and A.V. Ustinov, Phys. Rev. E 62, 2858 (2000).

[13] J.L. Ting and M. Peyrard, Phys. Rev. E 53, 1011 (1996).

[14] M. Peyrard, Europhys. Lett. 44, 271 (1998).
[15] A. Campa and A. Giansanti, J. Biol. Phys. 24, 141 (1999).

[16] M. Salerno and Y. Kivshar, Phys. Lett. A 193, 263 (1994).

[17] K. Baverstock, Int. J. Radiat. Biol. 47, 369 (1985).

[18] L. Yakushevich, Nonlinear Physics of DNA (John Wiley and Sons, New York, 1998).

[19] M. Peyrard and A.R. Bishop, Phys. Rev. Lett. 62, 2755 (1989).

[20] J. Sepulchre and R. MacKay, Physica D 113, 342 (1998).

[21] J.F.R. Archilla, R. MacKay, and J. Marín, Physica D 134, 406 (1999).

[22] G. Kopidakis and S. Aubry, Physica D 130, 155 (1999).

[23] K. Forinash, T. Cretegny, and M. Peyrard, Phys. Rev. E 55, 4740 (1997).

[24] Y.B. Gaididei, S.F. Mingaleev, and P.L. Christiansen, Phys. Rev. E 62, R53 (2000).

[25] P. Christiansen, Y.B. Gaididei, and S. Mingaleev, J. Phys.: Condens. Matter 13, 1181 (2001).

[26] R. MacKay and J. Sepulchre, Physica D 119, 148 (1998).

[27] J.M. Sanz-Serna and M.P. Calvo, Numerical Hamiltonian Problems (CRC Press, Boca Raton, 1993).

[28] J.F.R. Archilla, P.L. Christiansen, S.F. Mingaleev, and Y.B. Gaididei, J. Phys. A 34, 6363 (2001). 